\title{
Indoor/ outdoor navigation system based on possibilistic traversable area segmentation for visually impaired people
}

\author{
Jihen Frikha Elleuch, Dorra Sellami and Imen Khanfir Kallel \\ CEM Lab, National Engineering School of Sfax, 3038 Sfax, Tunisia
}

Received 13th October 2015; accepted 20th June 2016

\begin{abstract}
Autonomous collision avoidance for visually impaired people requires a specific processing for an accurate definition of traversable area. Processing of a real time image sequence for traversable area segmentation is mandatory. Low cost systems suggest use of poor quality cameras. However, low cost camera suffers from great variability of traversable area appearance at indoor as well as outdoor environments. Taking into account ambiguity affecting object and traversable area appearance induced by reflections, illumination variations, occlusions (, etc...), an accurate segmentation of traversable area in such conditions remains a challenge. Moreover, at indoor and outdoor environments, more added variability is induced. In this paper, we present a fast traversable area segmentation approach from image sequence recorded by a low-cost monocular camera for navigation system. Taking into account all kinds of variability in the image, we apply possibility theory for modeling information ambiguity. An efficient way of updating the traversable area model in each environment condition is to consider traversable area samples from the same processed image for building its possibility maps. Then fusing these maps allows making a fair model definition of the traversable area. Performance of the proposed system was evaluated on public databases, at indoor and outdoor environments. Experimental results show that this method is challenging leading to higher segmentation rates.
\end{abstract}

Key Words: Traversable area segmentation, indoor/outdoor environment, possibility theory, fusion, reference window, visually impaired people.

\section{Introduction}

In recent years, many electronic travel aids (ETA), especially vision-based obstacle avoidance systems, have been developed to help the visually impaired people to walk safely $[1,2]$. Vision- based obstacle detection is one of the major issues that need to be considered to ensure safe navigation of visually impaired people. The problem encountered in obstacle detection may often be reduced to a problem of traversable area segmentation. The state of the art of traversable area extraction for navigation assistance can be classified into two categories:

- The first category, where obstacle detection is carried out by considering depth information $[3,5,6$ and 7]: Accordingly, depth map is obtained from various capture devices, such as stereovision cameras [3, 4], or Kinect sensors [5, 6]. In [3], ground plane was estimated from stereo camera

Correspondence to: jihen_frikha4@yahoo.fr

Recommended for acceptance by < Giorgio Fumera $>$

http://dx.doi.org/10.5565/rev/elcvia. 818

ELCVIA ISSN: $1577-5097$

Published by Computer Vision Center / Universitat Autonoma de Barcelona, Barcelona, Spain 
image using RANSAC (RANdom SAmple Consensus) algorithm [4], combined with least-squares optimization. Object detection is performed by regrouping spatial neighbouring points above the ground plane. In [5], authors use the disparity map provided by the stereo camera, detects the ground plane applying a RANSAC algorithm in the v-disparity domain. In [6] authors use the same algorithm RANSAC is applied to depth map for determining the initial region of ground class, then a region growing method is applied to find similar colour regions. The depth map is provided by RGBD sensor. In [7], authors exploit the depth-maps given by a Kinect sensor to estimate the ground plane. After ground plane estimation, the objects in a region of interest are detected. The depth-map is divided in 3 vertical regions with 3 different orientations and a vibrating device is associated to each of them. Vibration intensity is stronger for the closer obstacle detected in each region.

The second category, where detection is based on appearance information such as texture, colour, hue and saturation for image segmentation using RGB camera [8-12]: In [8, 9], authors have been based on histogram features using hue and light information of the image. Accordingly, for each pixel, they compute the corresponding features and measure its similarity with the safe window. An empirical sub-threshold is then used for decision making. They are based on the assumption that the area, in front of the robot, is always traversable (initial state assumption). Then, they make use a given safe window to derive the properties of the surface to be analyzed. Such window will be relevant and a fair sample, taking into accounts the different environment conditions: luminance condition, ground texture and colour. However, given the pixel level dependency of this approach and eventual inaccuracy of the empirically chosen sub-threshold value, it cannot handle the condition variability of the image. Besides, it cannot be applied to a more general context such as, outdoor environment. Frikha et al. have improved in [10] the Ulrich method (described in [9]) to detect obstacles having the same colour as the traversable area by adding texture based features in segmentation [9]. This method can be easily applied in real time obstacle detection. Although it is efficient in indoor environment, it fails for outdoor context. In [11], Li and Birchfield propose gradient based techniques for traversable area segmentation. Unfortunately, such technique fails in some cases, due to lack of well defined boundaries. In [12], Kröse et al. propose the use of optical flow-based techniques; it solves the problem of complex background. However, it incurs additional computational cost and can be inaccurate in case of camera vibration and blurry image.

All these methods, when applied to outdoor/ indoor context, are subject to confusion in scene interpretation, induced by camera vibration, blurry ground edges, etc. Such ambiguity and uncertainty in representation should be considered, in data modelling for sake of robustness/ efficiency. Probability theory was the first model used to cope with value uncertainty problem, but they are not suitable to solve ambiguity categorizing problem [13]. Theories based on Fuzzy sets [14], proposed later on, by Zadeh, can model ambiguity but they fail in case of uncertain data. Theory of belief functions as well as possibility theory was proposed later on, to efficiently handle/model ambiguous/uncertain data [15]. However, we can encounter some difficulties in belief mass function estimation from histogram features. Possibility theory is however naturally adapted to segmentation problems based on histogram features. Exploitation of possibility theory is becoming, in latest years, more frequent in the frame of data processing, especially for uncertainty modelling. However, no previous work deals with traversable area segmentation, based on possibility theory.

We aim in this work at designing a powerful system that can be suitable for traversable area segmentation for different environment conditions, i.e. indoor, outdoor, and structured or not. We aim accordingly at proposing a global traversable area segmentation method. In this paper, we propose a possibility based segmentation method based on reference windows; we build an accurate possibility map allowing segmentation which is more adapted to the environment conditions. Hence, traversable area segmentation will be performed in indoor as well as outdoor environment.

The paper is organized as follows: in section 2, we detail the main contribution and describe different concerns of our system. In section 3, performance evaluations are presented. Finally, we draw in section 4 our conclusion. 


\section{Proposed segmentation system}

Our system is based on the following methodology aiming at extracting information from the video sequences to model traversable area for distinguishing it from non-traversable areas/obstacles. The main challenge is to propose an approach that is adaptable to variable environmental conditions. Figure 1 provides some examples of such challenging environmental conditions.
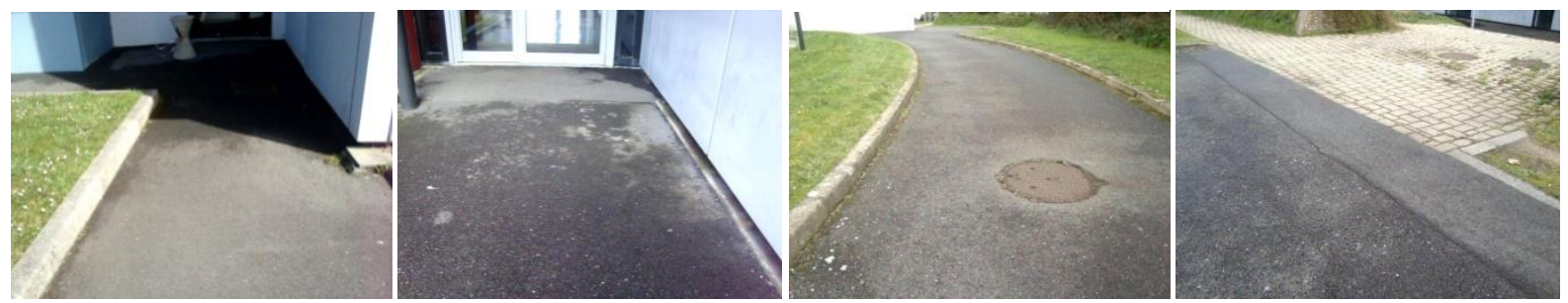

Fig.1: Examples of challenging environmental conditions with shadows, reflections from standing water and, wet prints and ground condition with existence of obstacle plane (sewer for example) in the traversable area.

The main target of the segmentation process is to assign each pixel from the analyzed image to a particular class of interest, namely traversable area and non traversable area. The image resulting from the pixel labeling is henceforth referred to as a thematic map. An important issue which can be addressed here concerns the fact that the segmentation lacks additional information related to the degree of certainty associated with each thematic class decision. In the proposed system, a pixel $X$ is described as a vector in an $N$ dimensional space:

$X=\left[x_{1}, x_{2}, \ldots, x_{n}\right], x_{n} \in S_{n}, n=1,2, \ldots, N$, where $S_{n}$ denotes the $\mathrm{n}^{\text {th }}$ knowledge source observation and $N$ is the number of sources. The universal set $S$ is the Cartesian product representing the multisource observation space.

If $C=\left[C_{1}, C_{2}, \ldots, C_{M}\right]$ is the set of $M$ image predefined classes, then, based on the fuzzy set theory, each class $C_{M}$ is defined as a fuzzy set over $S$ with $\mu_{X}\left(\mathrm{C}_{\mathrm{m}, \mathrm{n}}\right), m=1,2, \ldots, M$ denoting the membership degree with which the pixel $X \in S$ may be treated as belonging to the class $C_{M}$.

Possibility theory was introduced by Zadeh in 1978 [16], and developed by Dubois and Prade in 1988 [17]. This theory represents a tool allowing to model uncertainty related to imprecise information. The main purpose is to enable reasoning on imperfect information by the use of a possibility distribution $\pi(x)$. The possibility distribution $\pi(x)$ of a singleton $x \in \Omega$ is a mapping: $\pi: \Omega \rightarrow[0,1]$.

The possibility distribution of $x$ traduces a possibility value of its occurrence such as: $\pi(x)=1$ means that $x$ is completely possible and $\pi(x)=0$ means that $x$ is completely impossible. To characterize an event $A$, two measures are also defined: the possibility measure $\Pi(A)$ and the necessity measure $N(A)$. The possibility measure is defined as follows:

$$
\Pi(A)=\max _{x \in A} \pi(x)
$$

This measure shows the degree to which the event $A$ is possible on $\Omega$.

On the other hand, the necessity measure is defined as following:

$$
N(A)=1-\Pi(\bar{A})=1-\max _{x \in A}(1-\pi(x))
$$

Where $\bar{A}$ denotes the complement of $A$. This measure shows the degree to which $A$ is certain on $\Omega$ such as:

$N(A)=1$ means that $\bar{A}$ is impossible and then $A$ is completely certain and $N(A)=0$ means that $A$ is completely uncertain [18].

The proposed segmentation system, depicted in Figure 2, is an image segmentation system based on the concept of "possibilistic" knowledge propagation. Assuming the number of thematic classes contained in the 
analyzed image is given as a prior knowledge (corresponding to the closed world assumption in classification systems), the proposed system can be decomposed into the following sub-systems:

1. Generation of knowledge sources, which are here some sample windows taken as reference for class modeling.

2. Estimation of possibility distributions for each source of knowledge;

3. The fusion of these distributions in order to have a unique possibility map.

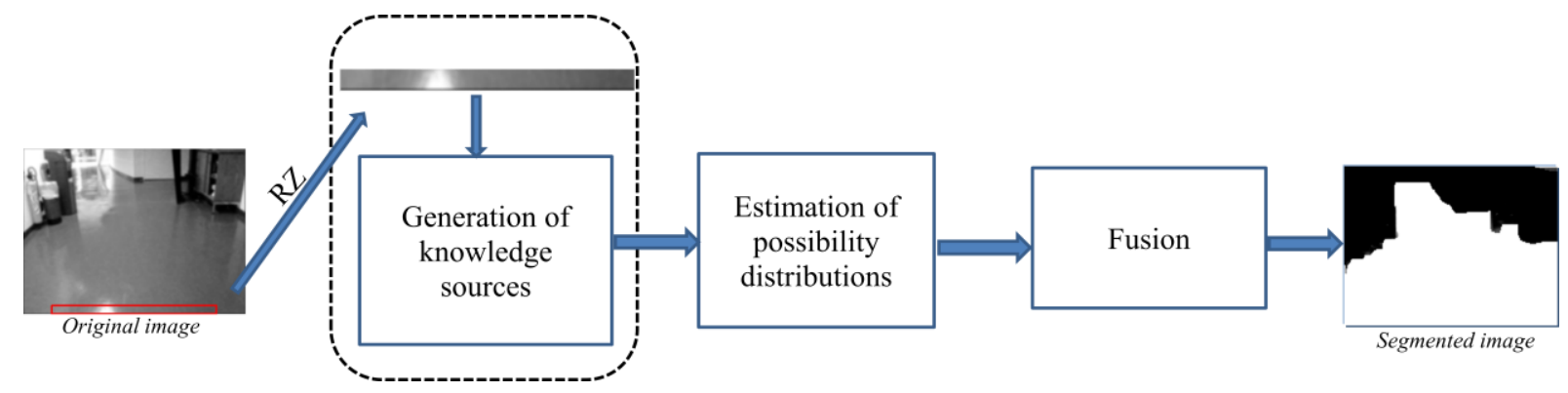

Fig.2: Basic steps of the proposed method for traversable area segmentation.

\subsection{Generation of knowledge sources}

In the first step, knowledge source generation is performed throughout extraction of some features related to the reference areas. Accordingly, colour input image RGB values are transformed to the HSV (Hue, Saturation and Value) colour space. The convenience of HSV space lies in the extremely intuitive manner of perceiving a colour. In [19], Suryani et al. have compared six colour spaces and have shown that the HSV space is more effective for image retrieval by content. From the input image, we then select three window samples of the traversable area class (RW1, RW2, and RW3), they will be called reference areas (e.g. green, blue and red area in Fig. 2). The size of each reference window is $25 \times 25$ pixels. For each one, we apply a mean linear spatial filtering with kernel size of $5 \times 5$ for both $\mathrm{H}$ and $\mathrm{S}$ channels.

\section{REFERENCE WINDOW DEFINITION:}

Several prior traversable area segmentation techniques have been developed around the assumption that the area immediately in front of the robot is initially traversable and thus they identify the pathway by comparison to a safe window near the bottom of the image $[8,9,14]$. This assumption is quite reasonable, given the orientation of the camera to the floor and since from the start of navigation, the system is assisting the visually impaired by avoiding obstacles. Thus, such region is the nearest to the visually impaired and cannot be nothing than an object free traversable area.

In our approach, we adopt this logic. Thus, the zone in front of the visually impaired person is used as reference one. Accordingly, for sake of generalization, we can be based on more than one reference window to model the traversable area. Furthermore, features extracted from these windows will be used to classify the whole pixels of the remaining image into traversable area or no traversable area. No traversable area means a potential obstacle.

In a preliminary work, we tried to optimize the choice of this reference window. 


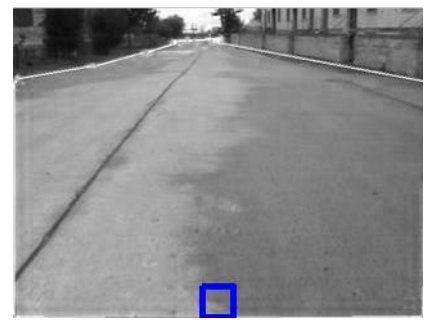

(a)

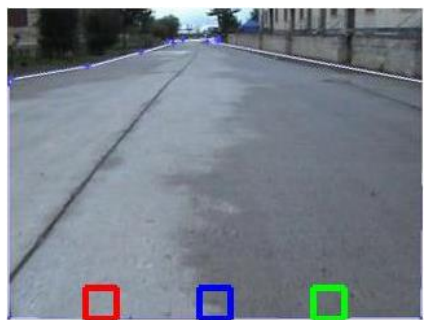

(b)

Fig.3: Reference window definition: (a) case of one window. (b) case of multiple windows.

Figure 3 illustrates two cases in which some reference windows are chosen. In Figure 3.a, a small central window in the bottom of the image is defined as a reference window with $25 \times 25$ pixels, which is assumed to be free from obstacles. This small area should represent the traversable area [14].

The robustness of our method relies on the adequate selection of this window to model appropriately the traversable area. In this example, we see that this single window is not so significantly illustrating the appearance of whole traversable area. Thus, to increase the reliability of the selection of this window, we will use additional small windows. This configuration is proposed to increase the number of samples describing the traversable area and to take into account more potential variability in the traversable area appearance. Hence, more precision will be reached in traversable area description.

\subsection{Estimation of possibility distributions}

In this step, each reference area $R W_{j}(j=1,2,3)$ is processed into three one-dimensional histograms $h_{j}$, for each channel ( $\mathrm{H}, \mathrm{S}$ and $\mathrm{V})$. We accordingly obtain for each image, nine histogram vectors.

Now, exploitation of data issued from the previous step is carried out in order to estimate the possibility map related to the traversable area class. The corresponding probability distribution $\mathrm{P}_{\mathrm{RWj}}$ will then be computed as the normalized histograms of the $\mathrm{RW}_{\mathrm{j}} \mathrm{HSV}$ channels.

It is good to know that there are many transformations to get possibility distributions from probability ones $[20 ; 21]$. These transformations should satisfy certain consistency principles, which are presented in several approaches in the literature [22,23]. We will study the best known approach which is the principle of consistency of Dubois and Prade [23] and we will use the symmetric transformation based on this principle.

Using the symmetric transformation of Dubois and Prade, these probability distributions will be converted into possibility distributions $\pi_{\mathrm{RWj}}$ [23]. This transformation keeps a good relationship between measure of probability and measure of possibility that conserve some characteristics, called consistency principle. In the following sub section, we will detail how we proceed to implement this transformation.

Given the possibility distributions corresponding to the three reference windows, classification of all pixels will be carried out by projecting their values on each possibility distribution. The obtained result will represent possibility maps, in which the possibility value of the class "traversable area" is equal to 1 . By applying fusion on these maps (each map is extracted from one reference window) using conjunction operator, a decision on pixel class will be performed. Such fusion is useful to remove ambiguities of one source through the information provided by other sources/additional knowledge. Let's remember that information is often imperfect in the decision aid systems. These imperfections are manifested in many forms: uncertainty, inaccuracy, incompleteness, ambiguity and conflict [23]. More detailed analysis is given in section 2.3 . 


\section{PROBABILITY-POSSIBILITY TRANSFORMATIONS}

- Consistency principle of Dubois and Prade

The consistency principle of Dubois and Prade suggests that what is probable should be possible. For instance, if $\mathrm{p}$ is the probability distribution and $\mathrm{P}$ is its measure, then any possibility distribution $\pi$, or any possibility measure $\Pi$, defined on a given domain $\Omega=\left\{\mathrm{w}_{1}, \ldots, \mathrm{w}_{\mathrm{i}}, \ldots, \mathrm{w}_{\mathrm{n}}\right\}$ will satisfy the following equation:

$$
\left\{\begin{array}{l}
\forall \mathrm{w}_{\mathrm{i}} \in \mathrm{A}, \pi\left(\mathrm{w}_{\mathrm{i}}\right) \geq p\left(\mathrm{w}_{\mathrm{i}}\right) \\
\forall A \subseteq \Omega, \Pi(\mathrm{A}) \geq P(\mathrm{~A})
\end{array}\right.
$$

Any possibility distribution satisfying the consistency principle can be seen as an upper bound of the probability distribution. In this case, we affirm that $\pi$ dominates $p$. In addition, equation (3) is based on the fact that the possibility representation is less informative than the probability one. Indeed, for the probability model, uncertainty is represented by a single value, while for the possibility model, uncertainty and imprecision are represented simultaneously by an interval [23].

- Transformation probability-possibility of Dubois and Prade.

Dubois and Prade defined a symmetric transformation $\mathrm{p} \rightarrow \pi$ satisfying the principle of consistency which is defined by [19] where $p_{i}=p\left(w_{i}\right)$ and $\pi_{i}=\pi\left(w_{i}\right)$ :

$$
\pi_{i}=\sum_{j=1}^{n} \min \left(p_{i}, p_{j}\right)
$$

By applying this method, we compute possibility distributions for each feature and for each sample of the "traversable area" class. Then, we obtain three possibility maps for each reference window:

From the possibility distribution and to make more reliable decision, we consider that only features, with possibility values greater than a predetermined threshold value Poss-th=0.2, can be considered safely as belonging to the traversable area. All similar features will be then classified as traversable area. Accordingly, the feature in the possibility distribution is discretized into a set of bins. For each query pixel, we compute similarity of its features with bin values, and the pixel inherits its class (traversable area or non traversable area) from the nearest similar bin. The bin step is then given by:

$$
\mathrm{F}_{\text {step }}=\frac{F_{\text {max }}-F_{\text {min }}}{\text { Number of bins }}
$$

Where $F_{\max }$ and $F_{\min }$ are respectively the maximum and the minimum values of the considered feature, which are adaptively computed for each image and the number of bins is chosen equal to 10 .

Figure 4 gives an illustrating possibility distribution of reference area $\mathrm{RW}_{1}$ (red one) corresponding to the V channel. 

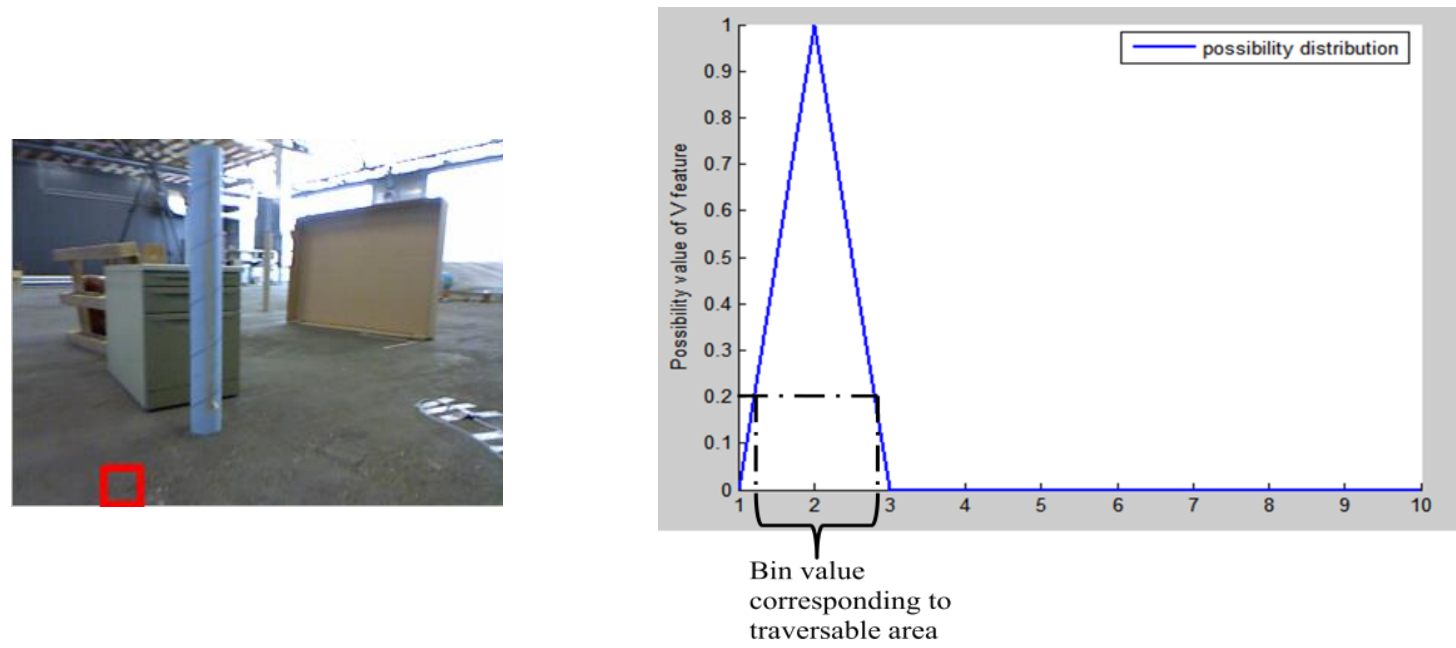

Fig.4: Possibility transformation of $V$ channel corresponding to red reference area

\subsection{Fusion of possibility maps}

The goal of the fusion step, in the proposed system, is to combine and exploit information for taking into account potential variability and data imperfections.

In the literature, three modes are conventionally distinguished for fusing information in possibility theory framework [24]:

- The conjunction: gathers the operators of intersection, this mode of combination must be used if measurements are coherent, i.e. without conflict. For example, "min" operator.

- The disjunction: gathers the operators of union; it must be used when measurements are in disagreement, i.e. in severe conflict. For example, "max" operator.

- The compromise: gathers the median operator or some average operators; it must be used when measurements are in partial conflict.

In the developed methods in our context, there are two steps in which we apply fusion:

- For each feature, possibility map was defined. This operation is reproduced to the three reference windows. After determining the different possibility maps for each channel, a first fusion is applied to combine possibility maps. "Max" operator is used in this first fusion in order to conserve maximal information corresponding to traversable area. Figure 5 gives an illustrating image describing construction of possibility map based on $\mathrm{V}$ channel. By repeating this procedure to $\mathrm{S}$ and $\mathrm{H}$ channels, two extra possibility maps will be constructed. Hence, at the end of this step, three possibility maps are defined $\Pi_{\mathrm{V}}, \Pi_{\mathrm{S}}$ and $\Pi_{\mathrm{H}}$. 


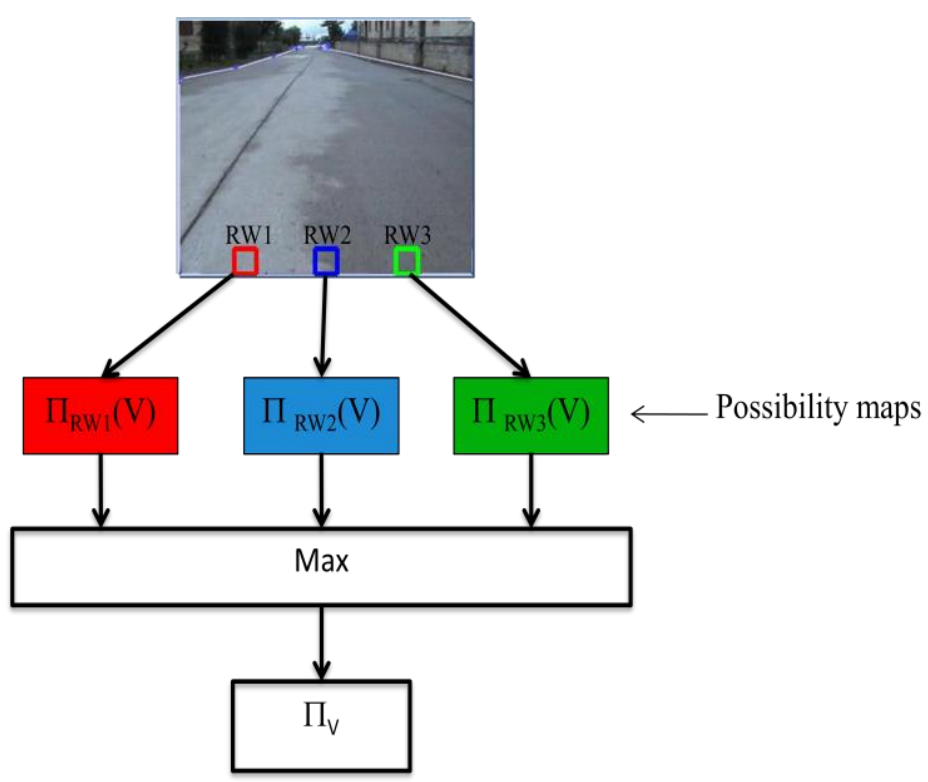

Fig.5: Possibility maps construction based on max operator (first fusion): case of $V$ channel.

- The second fusion is applied to combine the previous possibility maps i.e. $\Pi_{\mathrm{V}}, \Pi_{\mathrm{S}}$ and $\Pi_{\mathrm{H}}$. "Min" operator is used in this second fusion in order to define a unique possibility map.

In this step, a pixel that can be classified as traversable area is imperatively defined as traversable area in all $\Pi_{\mathrm{V}}, \Pi_{\mathrm{S}}$ and $\Pi_{\mathrm{H}}$ possibility maps. This fusion will be called decision step.

\section{Performance evaluation}

\subsection{Performance criteria}

To validate the proposed method and to explore its efficiency in detecting traversable area in several environments, we have performed a detailed evaluation based on a set of images from two databases: the first one deals with indoor context and the second one considers outdoor environment only. Accordingly, a set of 200 images corresponding to indoor environment were selected from the Technical University of Munich (TUM) dataset [25]. This dataset contains the color and depth images of a Microsoft Kinect sensor. The data was recorded at full frame rate $(30 \mathrm{~Hz})$ and sensor resolution $(640 \times 480)$. Then, from the second database, selection of 300 images corresponding to outdoor environment [26] is done, based on a video dataset comprising sequences captured under a wide range of environmental conditions and different ground types (cf. Figure 1). In each image, path and obstacle boundaries (ground truth) were manually drown.

The considered datasets are not acquired in the context of visually impaired people. However, the type of images and obstacles copes well with a visually impaired context.

Four cases were considered: homogenous environment, textured environment (shadow or wet print), obstacle almost confused with traversable area (due to its thin thickness) and textured traversable area.

The proposed approach deals with only software implementation solutions in Matlab environment (executed on a host computer with a processor Intel(R) Core(TM) i7-2.00 GHz with 4 Go of RAM). Accordingly, execution time allows processing of a video stream of 20 frames per second. We plan in future works to integrate this approach in our hardware platform.

To evaluate the proposed algorithm, the output was compared to the ground truth and different quality measures [27] were derived as follows: let TP be the number of true positive detections, FN be the number of false negative detections, TN be the number of true negative detections, and FP be the number of false positive detections. 
The Accuracy is the degree of closeness of measurements of a certain quantity to its actual value:

$$
\text { Accuracy }=\frac{T P+T N}{T N+F P+T P+F N}
$$

The Sensitivity can be defined as the percentage of true-positive results among those truly traversable areas (correct detections):

$$
\text { Sensitivity }=\frac{T P}{T P+F N}
$$

The Specificity can be defined by the percentage of true-negative results among the "non-traversable area" (correct rejections):

$$
\text { Specificity }=\frac{T N}{T N+F P}
$$

Missed detections (MD) is defined by:

$$
M D=\frac{F P}{T N+F P+T P+F N}
$$

False alarm (FA) is expressed as follows:

$$
F A=\frac{F N}{T P+F N}
$$

It is worth noting that a good detection corresponds on the one hand to high accuracy, high specificity and high sensitivity rates, and on the other hand, to lower FA and MD rates.

\subsection{Simulation results}

\section{SOME ILLUSTRATIONS OF THE WHOLE STEPS}

We discuss here the importance of the reference windows on the traversable area segmentation results (Figure 6). In fact, when dealing with the mean $\mathrm{H}$ image, we can easily notice that in some cases, there is no significant difference between maps issued from the red delineated window or the blue delineated window or the green delineated window. However, in the other cases (in ' $\mathrm{V}$ image' and ' $\mathrm{S}$ image), a big difference in the possibility map can be observed. Such result depends on the processed image, in fact when Saturation is almost constant in the image, then Hue channel and/or V can be more pertinent to reveal special data that can easily discriminate traversable area. 


\begin{tabular}{|c|c|c|c|}
\hline Channel & $\mathrm{V}(1)$ & $S(2)$ & $\mathrm{H}(3)$ \\
\hline $\begin{array}{l}\text { Original } \\
\text { image }\end{array}$ & & & \\
\hline (a) & & & \\
\hline (b) & & & \\
\hline (c) & & & \\
\hline (d) & & & \\
\hline
\end{tabular}

Fig.6: Illustration of different steps in the developed method. (a) Possibility maps based on red reference window, (b) Possibility maps based on blue reference window, (c) Possibility maps based on green reference window, (d) Possibility maps construction by applying fusion of previous primary possibility maps.

In Figure 6, we have defined the segmentation results as possibility maps for each channel $(\mathrm{H}, \mathrm{S}, \mathrm{V})$ and for each reference window. Hence, when applying the fusion for each channel, we take into accounts all data processed from the three reference windows. However, as we can notice visually, accuracy of the segmentation result at this step is weak ( $1^{\text {st }}$ Fusion in Fig.6d). Though, the fusion between the possibility maps processed from the three reference windows give better results (Figure 7). The adopted fusion operator used in this step is the conjunction one, intended for increasing the possibility that a pixel belongs to traversable area.

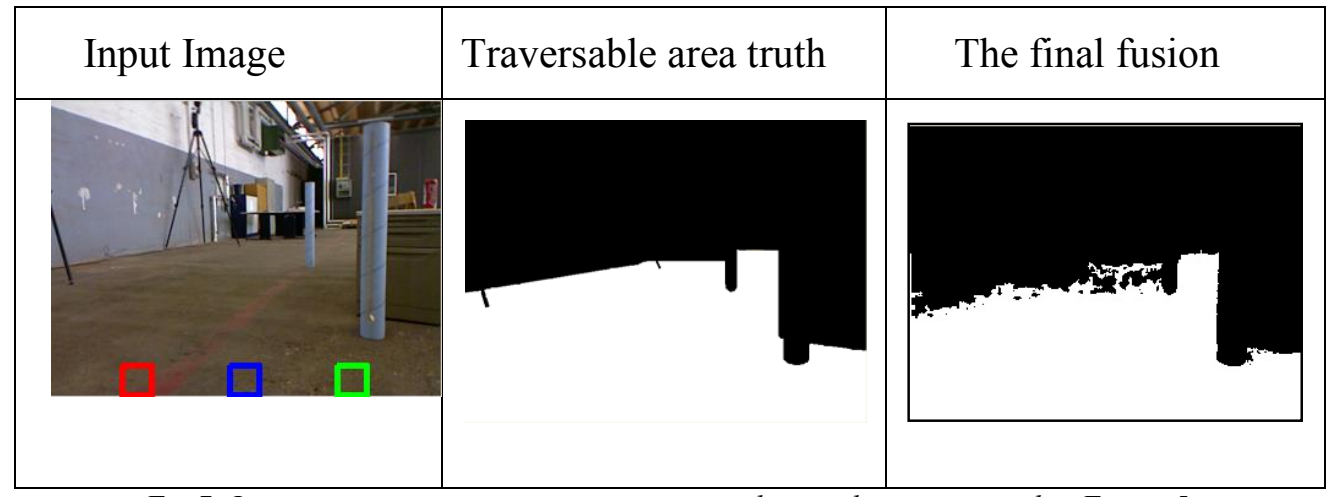

Fig.7: Image segmentation processes corresponding to the case treated in Figure 5. 


\section{STATISTICAL RESULTS}

Here, we undergo a fairer evaluation, based on the whole quantitative metrics defined in previous section, obtained in a statistical way from the images in the experimental sets. The results from the evaluation based on the testing image set are presented in Table.

\begin{tabular}{|c|c|c|c|c|c|c|}
\hline \multicolumn{2}{|c|}{ Challenge } & Accuracy & Sensitivity & Specificity & MD & FA \\
\hline \multicolumn{2}{|c|}{ Homogenous environment } & 0.941 & 0.959 & 0.935 & 0.047 & 0.040 \\
\hline \multirow{2}{*}{ Textured environment } & Shadow & 0.854 & 0.971 & 0.803 & 0.136 & 0.029 \\
\cline { 2 - 7 } & Wet print & 0.881 & 0.934 & 0.873 & 0.111 & 0.066 \\
\hline $\begin{array}{l}\text { Obstacles with thin thickness which is almost } \\
\text { confused with traversable area }\end{array}$ & 0.850 & 0.873 & 0.719 & 0.135 & 0.126 \\
\hline \multicolumn{2}{|c|}{ Textured traversable area } & 0.959 & 0.980 & 0.953 & 0.035 & 0.019 \\
\hline \multicolumn{2}{|c|}{ Average for all database } & $\mathbf{0 . 9 0 0}$ & $\mathbf{0 . 9 4 3}$ & $\mathbf{0 . 8 5 7}$ & $\mathbf{0 . 0 9 3}$ & $\mathbf{0 . 0 5 6}$ \\
\hline
\end{tabular}

Table 1: Different performance characteristics obtained by the processed possibility approach applied to different environment conditions as well as indoor/ outdoor type.

In the following, some significant image samples, illustrating each one particular challenge of our approach, were proposed (Figures 8, 9, 10 and 11). For all figures, three columns are used. In the first column, the input image is shown, whereas the second column contains the ground truth segmented image and in the last column, the processed image for traversable area segmentation is shown. For each environment condition, an example of indoor and outdoor environment cases is considered.

The first case (Figure 8) deals with traversable area segmentation in homogenous environment, i.e. where traversable area and non traversable area regions have almost identical appearance patterns. In such a case, there is good traversable area detection (sensitivity=0.959). The false alarms are also not very high $(\mathrm{FA}=0.040)$, which can be comfortable for the navigation of the blind people. On the other hand, it is very important to note that there is nearly no missed detection $(\mathrm{MD}=0.047)$. It means that no bad surprise for the blind people can be encountered when they navigate (all obstacles are detected) and thus increased safety.

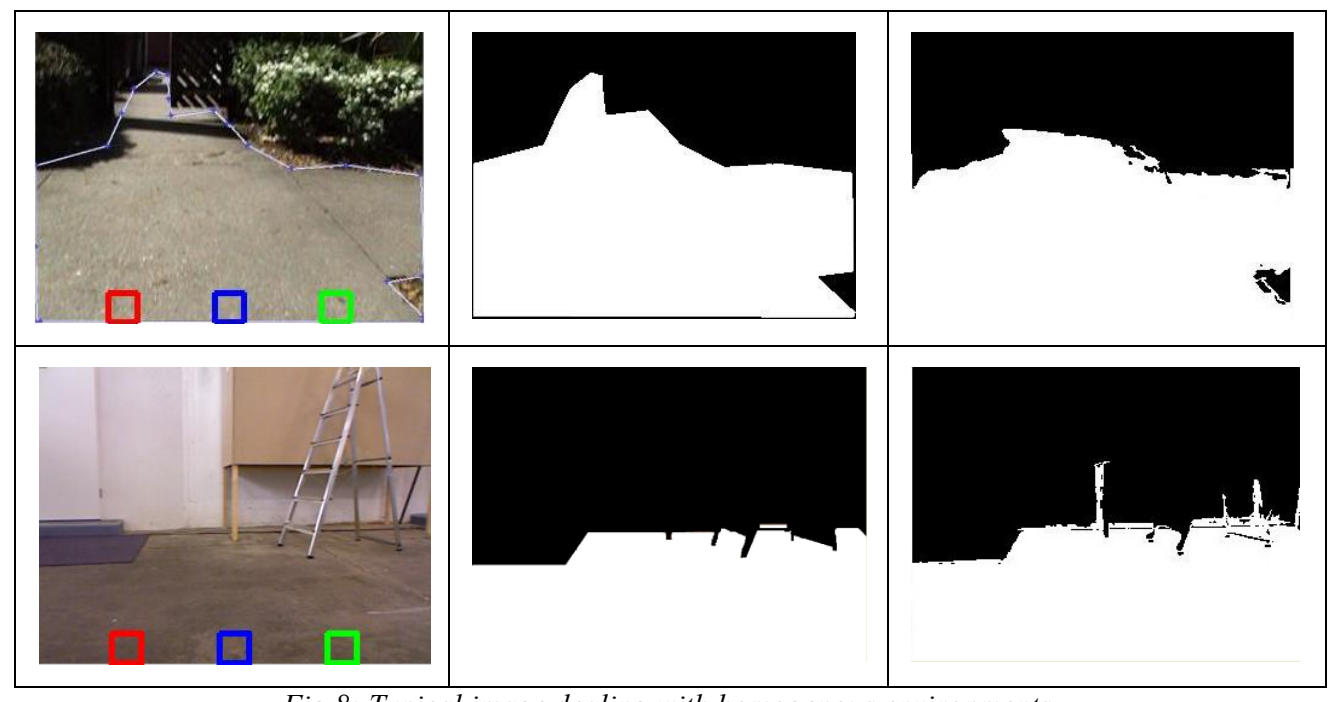

Fig.8: Typical image dealing with homogenous environments.

The second challenge is with respect to traversable area segmentation in the case of textured environment. This is illustrated in Figure 9; we can easily notice that the developed segmentation method is able to correctly classify the shadowed region. On the other hand, it is also able to solve highlight variation 
problems. The results show that the performance of the detection is better in the case of image with shadow compared to image with wet print. In fact, there is a reduction in number of false alarms (FA measure reduces from 0.066 to 0.029 ) and an increase in the number of correct detection (sensitivity increases from 0.934 to 0.971 ). Nevertheless, a small decrease in performance quality in the number of correct rejection (specificity decreases from 0.873 to 0.803 ) and missed detections is slightly higher as well (MD increases from 0.111 to 0.136 ).

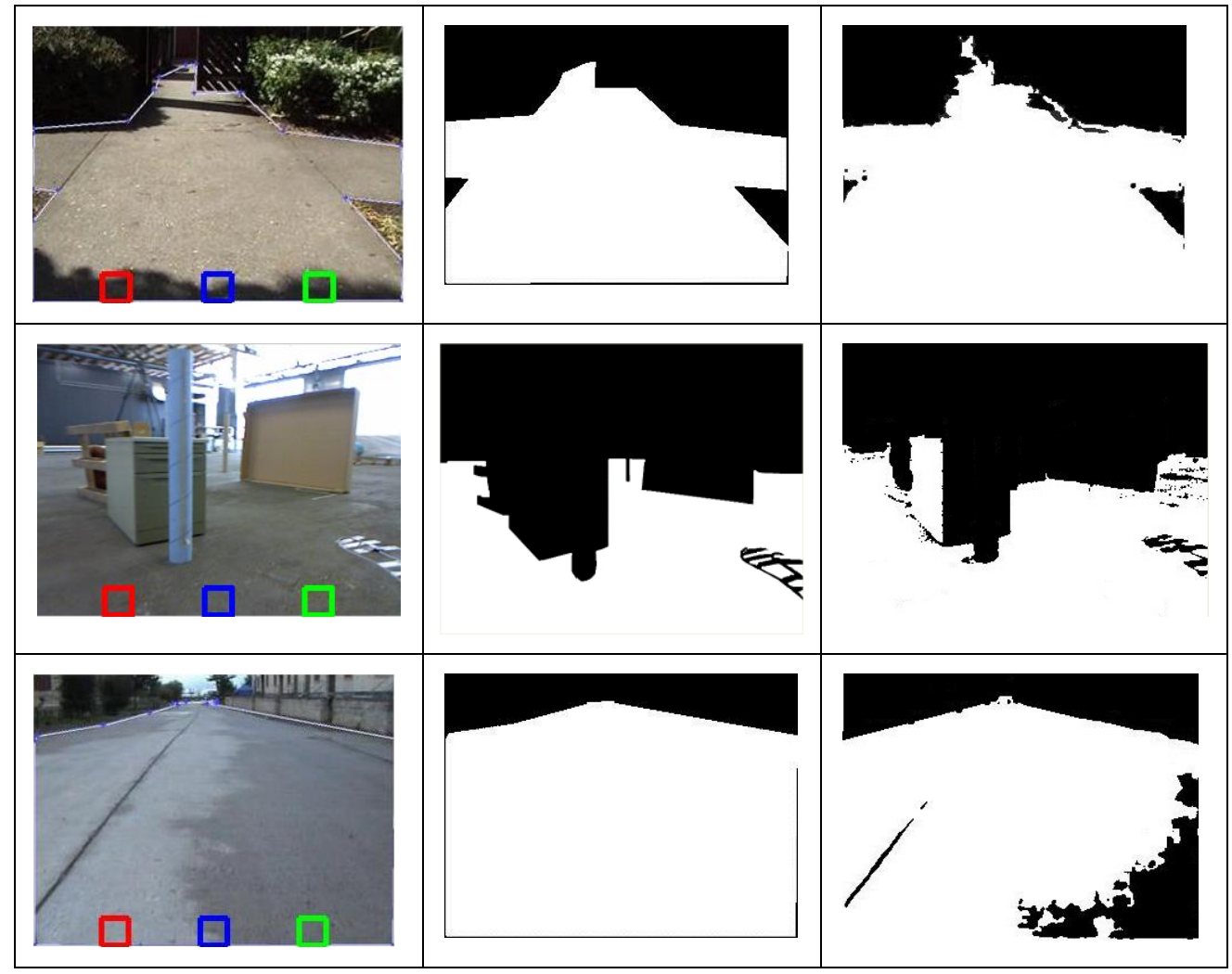

Fig.9: Typical image dealing with textured environment (shadow, highlight and wet print).

Concerning detection of obstacles with thin thickness, almost confused with traversable area, which is a crucial problem for blind auto-navigation indoor as well as outdoor, a significant improvement was achieved. Figure 10 illustrates the efficiency of our model since it succeeds to detect rigid obstacles with thin thickness. Actually, the sensitivity is 0.873 , the specificity is 0.719 and the accuracy is 0.799 . These values are too weak and seem to indicate bad traversable area detection. But in reality, this result is also induced by the worth definition of the ground truth (as found in the used database) which considers sometimes the rigid obstacle as traversable area. 


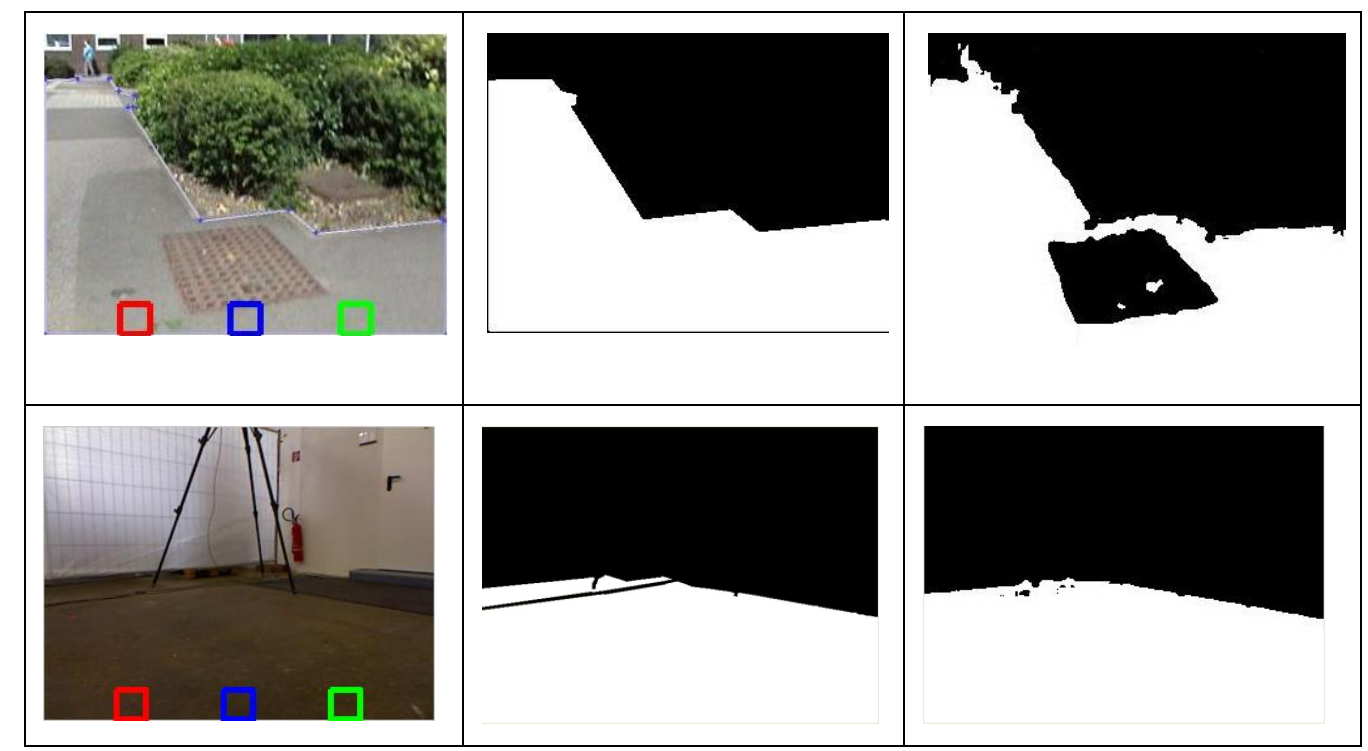

Fig.10: Typical images dealing with thin thickness detection which is almost confused with traversable area.

The last contribution deals with segmentation in textured ground. The obtained result is extremely interesting since there is no wrong classification of pixels belonging to traversable area. A typical example is shown in Figure 11, where a different traversable area texture is classified as traversable area and not as obstacle. The best measurement values of traversable area segmentation are found in this case $(0.959,0.980$ and 0.953 respectively for Accuracy, Sensitivity and Specificity). More specifically, false alarms (FA) and missed detection (MD) have also shown the lowest values (0.019 and 0.035 respectively). This means that navigation is simultaneously comfortable and safe.

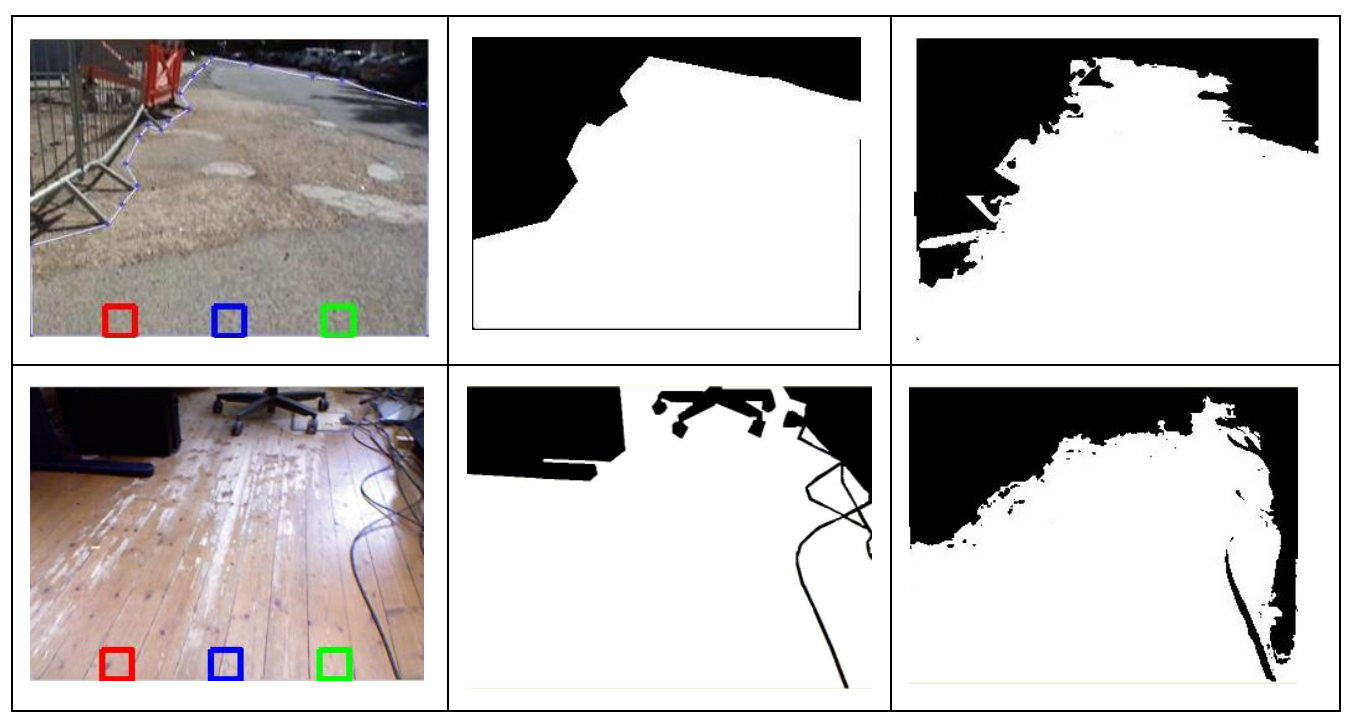

Fig.11: Typical image dealing with textured ground.

Table 2 illustrates comparison between the performances of the proposed possibility based method and the probability based approach. In the probability approach, the same segmentation procedure (considered in the possibility approach) is used without applying possibility transformation.

\begin{tabular}{|c|c|c|c|c|c|}
\hline Challenge & Accuracy & Sensitivity & Specificity & MD & FA \\
\hline Probability based approach & 0.730 & 0.796 & 0.669 & 0.219 & 0.162 \\
\hline Possibility based approach & 0.9 & $\mathbf{0 . 9 4 3}$ & $\mathbf{0 . 8 5 7}$ & $\mathbf{0 . 0 9 3}$ & $\mathbf{0 . 0 5 6}$ \\
\hline
\end{tabular}

Table 2: Comparison of performance characteristics obtained for probability and possibility approaches applied to different environment condition as well as indoor/ outdoor type. 
As table 2 shows, the proposed system outperforms the probability based approach in every test. In general, the probability based approach is usually quite poor for avoiding false alarms. Overall, the proposed system has an overall accuracy of over $90 \%$ in all these situations, while the other system can only achieve about $73 \%$.

The following illustrations (Figure 12) are with a good agreement with corresponding quantitative metric results (Table 2).

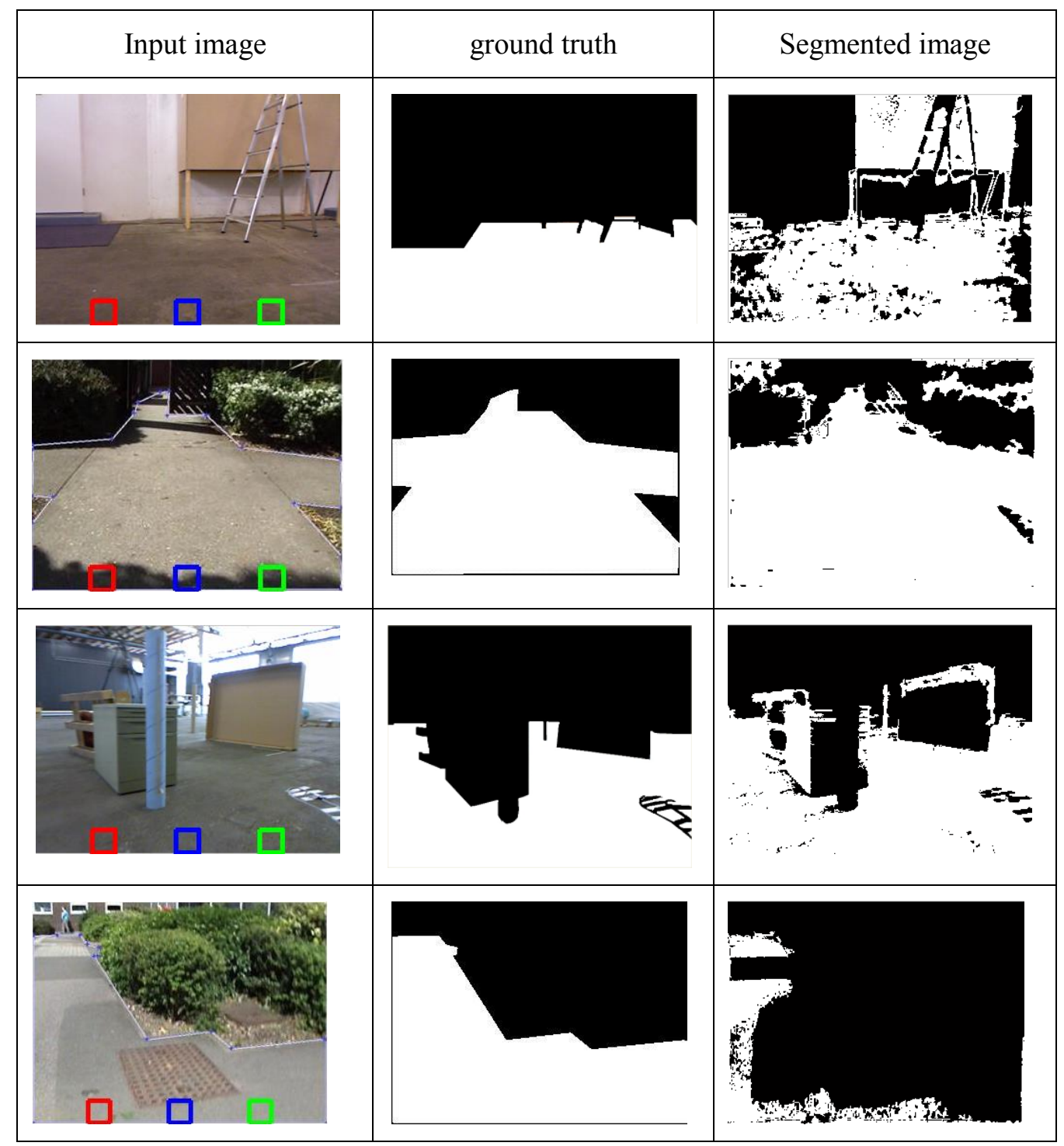

Fig.12: Image segmentation with probability based approach.

\section{3 comparison with conventional method}

In order to demonstrate the importance of the developed method a comparison with state of art is proposed. Two public dataset such as indoor [25] and outdoor environment [26] were used to achieve such comparison.

Aladren et al. [6] have proposed a system that detects and classifies the main structural elements of the scene providing the user with obstacle-free paths in order to navigate safely across unknown scenarios. This approach is based on RGB-D camera. Region growing method is applied to find same colour region. Initial region of ground class is defined throughout the RANSAC. Estimation of parameters based on RANSAC 
technique is very efficient it gives generally very good results even when there is image perturbation. However, to reach such result a very high execution time is needed ( 0.3 frame per second). Such computation time seems to be too high to ensure use of vision algorithms at a rate compatible with the navigation of the visually impaired people. The precision of this method is $99 \%$ (applied to indoor environment [25]).

A precision of $97 \%$ was achieved when applying the possibility based approach on this dataset [25]. The advantage of our method is the computation time which is 20 frames per second.

Katramados et al. [26] have proposed a real-time approach for traversable surface detection using monocular camera mounted on an autonomous vehicle. Detection of traversable area is performed by fusing color and texture information from HSL, YCbCr and $\mathrm{LAB}$ colour spaces. Histogram analysis is proposed to achieve image segmentation. The execution time is 25 frames per second. The mean accuracy of this method is $97 \%$ (applied on the outdoor environment [26]).

An accuracy of $98 \%$ was achieved when applying the possibility based approach on this dataset [26]. The computation time is 20 frames per second.

Table 3 illustrates comparison results with the above conventional methods.

In conclusion the possibility based approach is already suitable for indoor as well as for outdoor environments giving good segmentation quality with low execution time.

\begin{tabular}{|l|c|c|}
\hline performance & Frames/s & Precision \\
\hline Approach [6] with dataset1[25] & 0.3 & $99 \%$ \\
\hline Approach [26] with dataset2[26] & 25 & $97 \%$ \\
\hline Our approach with dataset1 & $\mathbf{2 0}$ & $\mathbf{9 7 \%}$ \\
\hline Our approach with dataset2 & $\mathbf{2 0}$ & $\mathbf{9 8} \%$ \\
\hline
\end{tabular}

Table 3: Comparison results with two state of the art approaches.

\section{Conclusions}

Ensuring safe auto-navigation of visually impaired people is a great challenge. Many traversable area segmentation methods were performed to achieve this goal. Efficiency of these methods must take into account real time application (short time response, reduced complexity for low cost implementation, easy use, etc). We have considered a context of a monocular based vision aid system mounted on electronic white cane. Very strict constraints, on the safety of the visually impaired user, lead us to the sake of a high reliable system. Segmentation of traversable area is a crucial task at indoor as well as outdoor environments. In that optic, we have to take into account some potential imperfections in the acquired image such as shadow, highlight, wet print, etc. For modeling such uncertainty, we apply the possibility theory. It's important to note that the success of this method can be explained by a good definition of the reference window, from which ground features were extracted. Typically, three reference windows were used to create the possibility model that defines the ground. Moreover, a fusion step, between computed possibility maps, is intended for more robust decision.

Results on two public databases prove the efficiency of the proposed approach. It ensures safe auto navigation of visually impaired people, since it solved many challenges such as: homogenous environment, obstacle with thin thickness, textured environment (shadow, highlight and wet print) and textured ground. Moreover, comparison with state of the art methods shows that our approach ensures an excellent trade-off between detection/accuracy performances and real time operations ability. In future works, some limitations such as motion blur in real-time implementations will be addressed. Finally, we project to integrate our software into an Arduino SD platform which can be mounted on a white cane. 


\section{References}

[1] D. Dakopoulos and N. G. Bourbakis; Wearable obstacle avoidance electronic travel aids for the blind: A survey, IEEE Transactions on System, Man and Cybernetics, vol. 40, no. 1, pp. 25-35, 2010. doi: 10.1109/TSMCC.2009.2021255.

[2] A. Sonda, F. Jihen, I. khanfir, and D. Sellami; New electronic cane for visually impaired people for obstacle detection and recognition, in Proceedings of the IEEE International Conference Vehicular Electronics and Safety, 2012, pp. 416 - 420. doi: 10.1109/ICVES.2012.6294266.

[3] T.Schwarze, M.Lauer, M.Schwaab, M. Romanovas, S. Böhm, and T.Jürgensohn; A camera-based mobility aid for visually impaired people, KI - Künstliche Intelligenz, vol.30, no.1, pp. 29-36, 2016. doi: 10.1007/s13218-015-04077.

[4] M. A. Fischler and R. C. Bolles; Random sample consensus: a paradigm for model fitting with applications to image analysis and automated cartography, Commun. ACM, vol. 24, no. 6, pp. 381-395, Jun. 1981. doi: $10.1145 / 358669.358692$.

[5] Stefano Mattoccia and Paolo Macr1; $3 D$ Glasses as Mobility Aid for Visually Impaired People, In Proc. Computer Vision - ECCV 2014 Workshops: Zurich, Switzerland, September 6-7 and 12, 2014. doi: 10.1007/978-3-31916199-0-38.

[6] A. Aladren, G. Lopez-Nicolas, L. Puig and J. J. Guerrero; Navigation Assistance for the Visually Impaired Using RGB-D Sensor With Range Expansion, IN IEEE Systems Journal, VOL 99, PP. 1-11, MAI 2014. doi: 10.1109/JSYST.2014.2320639

[7] F.L. M. Milotta, D.Allegra, F.Stanco, and G.M.Farinella; An Electronic Travel Aid to Assist Blind and Visually Impaired People to Avoid Obstacles, In Proc. Computer Analysis of Images and Patterns: 16th International Conference, CAIP 2015, Valletta, Malta, September 2-4, 2015. doi: 10.1007/978-3-319-23117-4-52.

[8] L.M. Lorigo, R.A. Brooks, and W.E.L. Grimson; Visually-guided obstacle avoidance in unstructured environments, IEEE/RSJ International Conference on Intelligent Robots and Systems, 1997, pp.373- 379. doi: 10.1109/IROS.1997.649086.

[9] I. Ulrich and I. Nourbakhsh; Appearance-based obstacle detection with monocular color vision, in Proceedings of the $12 \mathrm{~h}$ Conference on IAAI, 2000, pp. 866-871.

[10] J. Frikha, I. khanfir, and D. Sellami; New Ground Plane Segmentation Method for Electronic Cane, Journal of Image and Graphics Vol. 1, No. 2,pp.72-75, June 2013. doi: 10.12720/joig.1.2.72-75.

[11] Y. Li and S. Birchfield; Image-based segmentation of indoor corridor floors for a mobile robot, in IEEE/RSJ International Conference on Intelligent Robots and Systems, 2010, pp. 837-843. doi: 10.1109/IROS.2010.5652818.

[12] B.J.A Kröse, A. Dev, and F. C. A. Groen; Heading direction of a mobile robot from the optical flow, Image and Vision Computing, vol. 18, no. 5, pp. 415-424, 2000. doi: 10.1016/S0262-8856(99)00036-0.

[13] C. Dal Mutto, P. Zanuttigh, and G. Cortelazzo; Fusion of geometry and color information for scene segmentation, IEEE Journal of Selected Topics in Signal Processing, vol. 6, no. 5, pp. 505-521, 2012. doi: 10.1109/JSTSP.2012.2194474.

[14] Chieh-Li Chen, Yan-Fa Liao, and Chung-Li Tai; Image-to-MIDI mapping based on dynamic fuzzy color segmentation for visually impaired people, Pattern Recognition Letters, vol. 32, no. 4, pp. 549- 560, 2011. doi:10.1016/j.patrec.2010.11.019.

[15] P. Xu, F. Davoine, J.B. Bordes and T. Denoeux ; Fusion d'informations pour la compréhension de scènes, Traitement du signal, no 1-2, pp.57-80,2014. doi : 10.3166/TS.00.9-31.

[16] L. A. Zadeh; Fuzzy Sets as a Basis for a Theory of Possibility Fuzzy Sets and Systems, Elsevier, pp. 3-28,1978. 
[17] D .Dubois, H. Prade; Théorie des possibilités, applications à la représentation des connaissances en informatique, Masson, Paris, 1988.

[18] W. Eziddin, J. Montagner and B. Solaiman; An Iterative Possibilistic Image Segmentation System: Application to breast cancer detection, 13th Conference on Information Fusion (FUSION), 26-29 July 2010. doi: 10.1109/ICIF.2010.5712098.

[19] L. Suryani, and L. Guojin; Effectiveness and efficiency of six color spaces for content based image retrieval, in Proceedings of the International Workshop on Content-Based Multimedia Indexing (CBMI), 2003.

[20] D. Dubois and H. Prade; A set-theoretic view of belief functions: logical operations and approximations by fuzzy sets, International Journal of General Systems, vol.12, pp.193-226, 1986. doi: 10.1007/978-3-540-44792-4_14.

[21] M. Oussalah; On the probability/possibility transformations: a comparative Analysis, International Journal of General Systems, vol.29, no.5, 671-718, 2000. doi: 10.1080/03081070008960969.

[22] Mouchaweh M.S., Bouguelid M.S., Billaudel P. and Riera B; Variable Probability-possibility Transformation, 25th European Annual Conference on Human Decision-Making and Manual Control (EAM'06), 2006.

[23] D. Dubois and H. Prade; On possibility/probability transformations, Fuzzy Logic, pp. 103-112, 1993. doi: 10.1007/978-94-011-2014-2_10.

[24] H.J. Zimmermann; Fuzzy Sets Theory and its Applications, 2nd ed. Boston: Kluwer Academic Publishers, 1991. doi: 10.1007/978-94-015-7949-0.

[25] J. Sturm (2014) computer vision group homepage. [Online] Available:

http://vision.in.tum.de/data/datasets/rgbd-dataset.

[26] I. Katramados, S. Crumpler, and T.P. Breckon; Real-time traversable surface detection by colour space fusion and temporal analysis, In Proc. International Conference on Computer Vision Systems, 2009. doi: 10.1007/978-3-64204667-4_27.

[27] Matthias Kohl; Performance Measures in Binary Classification, International Journal of Statistics in Medical Research, 2012, 1, 79-81. 